\title{
INTELIGENCIA Y APRENDIZAJE: DEL ÁMBITO DOCENTE AL CONTEXTO ORGANIZACIONAL
}

PH.D. Rafael Bell Rodríguez Vcerrector Académico del Instituto

Superior Tecnológico de Formación rafael.bell@formación.edu.ec
PH.D. Belinda Lema Cachinell Rectora del Instituto Superior Tecnológico de Formación martalema@formacion.edu.ec
PH.D.(a). Alejandro Lema Cachinell

Director de Investigación Instituto Superior

Tecnológico de Formación alejandrol@formacion.edu.ec

Palabras claves: inteligencia, aprendizaje, organizaciones, aprendizaje organizacional

Recibido: 02 de Junio del 2016

Keywords: intelligence, learning, organizations,

Aceptado: 25 de Julio del 2016

organizational learning

\section{RESUMEN}

La inteligencia, el aprendizaje y su interrelación constituyen uno de los elementos fundamentales del proceso de enseñanza -aprendizaje. Sin embargo, su alcance va más allá del contexto educativo y está comenzando a desempeñar un rol determinante en el análisis del desarrollo de las organizaciones. En ese sentido, en el presente artículo se presentan diferentes puntos de vistas relacionados con la comprensión de la inteligencia y el aprendizaje organizacional. De la misma forma, se presentan evidencias y resultados que muestran la necesidad de su aplicación en el contexto organizacional. Tal el caso del Instituto Superior Tecnológico de Formación

\section{ABSTRACT}

Intelligence, learning, and its interaction constitute one the cornerstone in the teaching process. However, its scope goes beyond the teaching context to begin playing an important role in the analysis of the development of the organizations. In that sense, different points of view concerning the comprehension of intelligence and organizational learning are presented in this article. Furthermore, evidences and results showing the need of its application in the organizational context are included in the paper; this is the particular example of the Instituto Superior Tecnológico de Formación Profesional Administrativa y Comercial of Guayaquil. 


\section{INTRODUCCIÓN}

La preocupación por la comprensión de la inteligencia y su relación con el aprendizaje se ha convertido en un tema de privilegiada atención en las investigaciones de psicólogos, neurólogos, pedagogos, lingüistas, docentes y otros especialistas, que aúnan sus esfuerzos con el ánimo de revelar los procesos, vías y mecanismos, muchas veces ocultos, que posibilitan el éxito de los seres humanos en el enfrentamiento de los disímiles retos a los que se enfrentan en las múltiples facetas de la vida diaria.

Comúnmente se tiende a asociar los conceptos de inteligencia y aprendizaje con el ámbito docente, en concreto con la labor de las escuelas y con la actividad de profesores y estudiantes, existiendo también una cierta inclinación hacia su comprensión a nivel individual. Sin embargo, el alcance de estos conceptos rebasa los límites señalados y encuentra en las organizaciones un escenario propicio para su introducción y aplicación de manera creativa.

Derivado de ello el objetivo del presente artículo consiste en analizar algunas de las principales aristas en la comprensión de la inteligencia y del aprendizaje en el contexto organizacional con el ánimo de estimular la reflexión y el debate en torno a sus contenidos e implicaciones para las organizaciones, en concreto para el Instituto Superior Tecnológico de Formación Profesional Administrativa y Comercial de Guayaquil, Ecuador.

\section{DESARROLLO}

La investigación se desarrolló bajo un enfoque eminentemente cualitativo, apoyada en el uso de métodos de nivel teórico y empírico. Como métodos teóricos se utilizaron los siguientes:

Analítico-sintético: Para el estudio de las diversas fuentes bibliográficas consultadas acerca del objeto de esta investigación, en concreto de las teorías y los conceptos referidos a la inteligencia y al aprendizaje, incluidos los relacionados con el ámbito organizacional. .

Inductivo-deductivo: Como opción para procesar y valorar la información particular y general consultada, extraer conclusiones y contrastarla con los propios procesos de desarrollo de la organización.

Histórico-lógico: Con la finalidad de abordar la evolución histórica de la comprensión de la inteligencia y su vínculo con el aprendizaje.

Los métodos empíricos utilizados fueron la observación participante y el estudio biográfico narrativo. Mediante la observación se describieron los elementos comunes de la cultura de la organización, en este caso del Instituto Superior Tecnológico de Formación Profesional Administrativa y Comercial y las condiciones y rasgos que permitirían caracterizar a esta institución como una organización inteligente.

La aplicación del estudio biográfico narrativo permitió una mejor comprensión de las relaciones humanas, tensiones, limitaciones y potencialidades de la organización y su capacidad para la continuidad de su aprendizaje, estimulada en estos momentos por el logro de la condición de centro acreditado como resultado de la evaluación aplicada por el Consejo de Evaluación, Acreditación y Aseguramiento de la Calidad de la Educación Superior 
(CEAACES) del Ecuador.

\section{RESULTADOS}

\section{A) Comprensión de la inteligencia y sus diferentes tipos.}

Una aproximación inicial a los conceptos que motivaron el desarrollo de esta investigación puede ser realizada mediante la búsqueda de información en internet que, según el buscador que se utilice, arrojará una cantidad diferente de resultados, pero en todos los casos, se obtendrán cantidades millonarias, superiores a los 17 millones de registros, lo que es una clara evidencia de la abundancia de información existente al respecto y de la diversidad de posturas y enfoques que las mismas reflejan.

En el caso de la inteligencia, en el amplio volumen de información consultada, se evidencia la trayectoria seguida por este concepto en su evolución, marcada por la concentración de la atención en la dimensión individual de la inteligencia y en su medición, generalmente asociada a los resultados de los sujetos que eran objeto de evaluación en el cumplimiento de tareas relacionadas con las áreas de lengua y matemáticas, lo que, de una u otra manera, terminaba por relacionarse con la escuela.

Los aspectos esbozados constituyen el punto de partida de las concepciones actuales acerca de la inteligencia, que se distinguen por el tránsito desde una concepción unitaria de la inteligencia, asociada a los conceptos de cociente intelectual y edad mental, hacia su comprensión multifactorial con un creciente reconocimiento del papel de los factores ambientales y culturales en su desarrollo (Gardner, 1983).

En este contexto en la investigación desarrollada se resaltan los estudios realizados por el investigador norteamericano Howard Gardner (1995) que lo condujeron a la presentación de su teoría sobre las inteligencias múltiples, uno de los pilares en los que se sustenta la investigación que se presenta, que asume los aportes de Gardner (1995) como los generadores de una importante transformación en relación con la concepción de la inteligencia, propiciando la superación de su visión estática, monolítica y prácticamente invariable.

Desde esa perspectiva, Gardner (1995) señala que la inteligencia está determinada por la capacidad para la solución de determinados problemas o para la creación de nuevos productos, que han de mostrar su valor en los diferentes contextos culturales en los que el individuo se desenvuelve.

En su teoría, este autor define los siguientes tipos de inteligencia: lingüística, lógico-matemática, espacial, cinético-corporal, musical, interpersonal, intrapersonal y naturalista.

El análisis de las características e implicaciones de los mencionados tipos de inteligencia ha sido objeto de atención en múltiples investigaciones y estudios, por lo que en esta ocasión no se consideró imprescindible profundizar en este tema. Sin embargo, vale la pena apuntar que la teoría de las inteligencias múltiples no es una propuesta acabada, lo que se evidencia en la labor de su propio creador y de otros investigadores, que continúan promoviendo el desarrollo de nuevas investigaciones que les permitan la mejor comprensión y aplicación de los tipos de inteligencia identificados hasta el momento y la posible determinación de nuevos tipos de inteligencia. 


\section{B) Inteligencias y mundo empresarial}

En consonancia con lo antes señalado la investigación permitió constatar el creciente reconocimiento que alcanza el valor de los diferentes tipos de inteligencia para la actividad empresarial y el desarrollo, en ese contexto, de propuestas que sugieren el incremento de los tipos de inteligencia inicialmente establecidos con la incorporación de otros tipos de inteligencia de particular significación para el mundo empresarial y entre los que se incluyen, de acuerdo con Valdez, Novoa y Huerta (2012) la denominada inteligencia organizativa y la inteligencia organizacional, a las que convendría añadir la inteligencia competitiva (Morcillo, 2003).

Según Valdez, Novoa y Huerta (2012) la inteligencia organizativa puede ser definida de la siguiente manera:

Capacidad del ser humano de organizar ideas, pensamientos, cosas y personas dando coherencia, sustento, dirección y orden a las diferentes acciones que realiza, a través del tiempo y del espacio, con el objetivo de alcanzar ciertos objetivos y metas previamente establecidas (p.15).

Para estos mismos autores las seis áreas que configuran este tipo de inteligencia son las siguientes:

a) Organización de ideas, pensamientos, emociones y sentimientos.

b) Organización de personas, grupos, equipos de trabajo y organizaciones.

c) Organización de tiempos.

d) Organización de espacios.

e) Organización de objetos y cosas.

f) Organización de acciones, planes y proyectos.

Como todos los tipos de inteligencia, la inteligencia organizativa recorre un largo camino para su desarrollo, y en su evolución, la experiencia práctica juega un determinante papel.

En cuanto al análisis y la comprensión de la inteligencia organizacional, se revela una situación similar a la existente con la inteligencia humana, caracterizada por la existencia de diversidad de enfoques, que dependen fundamentalmente de los énfasis que se colocan en diferentes aspectos, lo que permite identificar, entre los principales planteamientos, los siguientes:

- $\quad$ Comprensión de la inteligencia organizacional como capacidad

- Definición de la inteligencia organizacional como un proceso y como un producto

- $\quad$ Establecimiento de la inteligencia organizacional como una función sistémica

Así y asumiendo como criterio rector el referido al desarrollo de las capacidades, Lozano y Hernán (2014), definen la inteligencia organizacional como la capacidad de las organi- 
zacional como la capacidad de las organizaciones para el autoaprendizaje, para la creación y gestión del conocimiento con el propósito de aplicarlo con un enfoque estratégico y de responder a los cambios y retos del entorno.

La colocación de las capacidades como criterio de partida de las definiciones de inteligencia esbozadas se convierte en una importante premisa para acometer las estrategias que conduzcan a su mejor desarrollo tanto desde el punto de vista individual como colectivo.

A tal fin, la investigación permitió resaltar el carácter dinámico de las capacidades, cuyas potencialidades de desarrollo están íntimamente relacionadas con la influencia de los factores sociales y culturales. En ese contexto el aprendizaje está llamado a jugar un determinante papel para el logro de organizaciones inteligentes, como se refleja en el siguiente apartado.

\section{C) Organizaciones inteligentes}

Al hilo de lo hasta aquí señalado no es difícil advertir el reto que representa definir el carácter inteligente de una organización, pues existen al respecto diversos puntos de vista y criterios de los investigadores, quienes difieren en relación con los aspectos que consideran claves para arribar a tal conclusión.

En el centro de las referidas diferencias se identificaron diversos factores y condiciones entre los cuales se distinguen la motivación laboral, el clima de la organización, el aprovechamiento consciente de los medios para acceder, adquirir, almacenar y compartir información (Danger, 2005) a los que se unen la capacidad de integración y el establecimiento de una sólida relación entre el conocimiento y la toma de decisiones y el vínculo entre la difusión del conocimiento y el cumplimiento de los objetivos de la organización.

A la luz de los aspectos expuestos, el desarrollo de la investigación permitió establecer los puntos de coincidencia con las posiciones de León, Tejada y Yataco (2003) quienes precisan que las organizaciones inteligentes son "aquellas capaces de aprender permitiendo así expandir sus posibilidades de crecimiento" (p.82). Al mismo tiempo se subraya que una empresa inteligente es la que es capaz de desarrollar todas sus potencialidades con el fin de maximizar y fortalecer la capacidad de aprendizaje de todos sus equipos tanto a nivel individual como colectivo, lo que ha de favorecer el logro de los resultados propuestos y el fomento de nuevos modos de pensar y de actuar (Lanzas, Lanzas y Arias, 2005).

Derivado de lo antes señalado es posible destacar determinados aspectos que pueden ser considerados como factores comunes que han de caracterizar a las organizaciones inteligentes, los que de acuerdo con Gil (2006) pueden ser expresados de la siguiente manera:

- $\quad$ Proactividad en la generación de cambios y en la búsqueda de mejores resultados.

- Interacción entre el desarrollo individual y el desarrollo colectivo

- $\quad$ Promoción del aprendizaje

- Establecimiento del conocimiento como factor fundamental para la producción

- $\quad$ Relación entre las funciones de pensar y de hacer 
- Transformación de los sistemas de información en sistemas de comunicación

Lógicamente, para que una empresa se transforme en una organización inteligente debe desarrollar su capacidad para aprender individual y colectivamente lo que presupone, entre otros factores, la integración de equipos de trabajo con disposición para el intercambio, la interacción, la actividad conjunta y el trabajo dirigido a la generación de vías, mecanismos y procedimientos que favorezcan la búsqueda, el dominio, aprovechamiento y la difusión del conocimiento (Gil, 2006).

Por tanto, una organización que aprende, como señala Martín (2007) se gesta a partir de sus equipos de trabajo y ha de orientar su labor hacia el óptimo aprovechamiento del talento humano. En este sentido, la flexibilidad, la tensión creativa y el grado de compromiso de los empleados con la cultura de la empresa deben convertirse, junto con la calidad, la eficacia y su constante mejoramiento, en sus características más relevantes.

Consiguientemente se puede afirmar que la transformación de una empresa en una organización inteligente representa un complejo proceso en cuyo desarrollo se deben superar múltiples obstáculos, entre los cuales pueden ser destacados, de acuerdo con Martín (2007) los siguientes:

- $\quad$ Persistencia de hábitos y estereotipos que obstaculizan las transformaciones requeridas al limitar la participación, el intercambio y la colaboración mutua.

- Dificultades individuales para discriminar la información de mayor relevancia.

- Insuficiente reconocimiento real del valor del conocimiento y subestimación de las potencialidades del aprendizaje organizacional.

Es evidente que la superación de los obstáculos señalados demanda de las organizaciones la movilización de sus recursos y el máximo aprovechamiento de sus reservas culturales, específicamente de aquellas llamadas a estimular el reconocimiento y desarrollo del aprendizaje organizacional, de cuya determinante contribución depende en gran medida el logro de una organización inteligente.

\section{C) ITF: una organización que aprende}

Una organización inteligente es, en buena medida, la expresión del resultado del aprendizaje organizacional, comprendido, como justamente apuntan García, Jiménez y Lloréns (2011) como el proceso orientado al mantenimiento y mejoramiento del desempeño de la organización, en cuyo contexto su experiencia alcanza un valor de gran relevancia.

En ese sentido la investigación desarrollada permitió revelar cómo el aprendizaje organizacional se va convirtiendo en la opción estratégica del ITF para enfrentar con agilidad y efectividad los retos que se derivan de las nuevas condiciones presentes en el medio interno y en el entorno.

En la experiencia del Instituto de Formación se identificaron los siguientes factores claves para la promoción del aprendizaje organizacional: 
Los colaboradores (las personas)

El trabajo en equipo

La comunicación fluida

El liderazgo

El clima y ambiente institucional

Sobre este último factor la investigación encontró hallazgos interesantes, corroborando lo reflejado al respecto en el informe del CEAACES en el que se precisa que en la evaluación del ambiente institucional, al igual que en otros indicadores, el Instituto alcanza una valoración que supera el valor estándar de calidad.

Al mismo tiempo la investigación aportó evidencias en relación con las capacidades individuales y colectivas que influyen tanto a nivel grupal como individual en la promoción del aprendizaje organizacional.

Desde el punto de vista individual un importante papel lo desempeña el domino personal, que se encuentra íntimamente relacionado con la inteligencia intrapersonal e interpersonal mientras que a nivel colectivo sobresalen la conformación de una imagen compartida del futuro de la organización y los deseos de seguir creciendo juntos en el sentido humano y profesional.

\section{CONCLUSIONES}

El análisis de algunas de las principales aristas en la comprensión de la inteligencia y del aprendizaje en el contexto organizacional y el reflejo de su concreción en el ITF ha permitido la sistematización de un marco conceptual y la identificación de los factores que permiten definir al ITF como una organización que aprende. En consecuencia, la investigación constituye una nueva evidencia de la necesidad y conveniencia de continuar incorporando con creatividad conceptos como inteligencia y aprendizaje al ámbito organizacional, rebasando los límites habituales de su uso asociados al entorno docente. 


\section{REFERENCIAS BIBLIOGRÁFICAS}

CEAACES (2016). Informe Final de la Evaluación del Entorno de Aprendizaje de los Institutos Superiores Técnicos y Tecnológicos. Quito: Dirección de Evaluación y Acreditación de Institutos Superiores.

Danger, Y. (2005). ¿Qué le impide a su empresa convertirse en una organización inteligente? Sociedad y Economía, 108, 554-569.

García, V., Jiménez, M. y Lloréns, F. (2011). Influencia del nivel de aprendizaje en la innovación y desempeño organizativo: factores impulsores del aprendizaje. Revista Europea de Dirección y Economía de la Empresa, 20(1), 161-186.

Gardner, H. (1995). Inteligencias múltiples: la teoría en la práctica. Barcelona: Paidós.

Gil, J.J. (2006). La gestión empresarial bajo el enfoque de las organizaciones inteligentes en la sociedad de la información. Revista Negotium/Ciencias Gerenciales, 2(6), 33-54.

Lanzas, A.M., Lanzas, V. E. y Arias, L. (2005). Creación de empresas inteligentes, cinco disciplinas que contribuyen al proceso. Scientia et Technica, XI (28), 151-154.

León, R., Tejada, E. y Yataco, M. (2003). Las organizaciones inteligentes. Industrial data, $6(2), 82-87$.

Lozano, J. \& Hernán, C. (2014). Una propuesta para la definición de la inteligencia organizacional. Universidad \& Empresa, 16(26), 159-175.

Martín, I. (2007). Retos de la comunicación corporativa en la sociedad del conocimiento: de la gestión de información a la creación de conocimiento organizacional. Signo y Pensamiento, 51 (XXVI), 52-67

Morcillo, P. (2003). Vigilancia e inteligencia competitiva: fundamentos e implicaciones. Revista madri+ d, (17), 2.

Valdez, A., Novoa, J. y Huerta, D. (2012). Inteligencia organizativa y liderazgo organizacional. INCEPTUM, VII (12), 11 - 23. 